\title{
Construction of Adaptive Consensus Gains for Multiagent Systems with Multiplicative Noise
}

\author{
Shoubo Jin (i) ${ }^{1}$ and Qiuju Yu (iD) ${ }^{1,2}$ \\ ${ }^{1}$ School of Mathematics and Statistics, Suzhou University, Suzhou, Anhui, China \\ ${ }^{2}$ School of Mathematical Sciences, Universiti Sains Malaysia, Gelugor, Penang, Malaysia \\ Correspondence should be addressed to Shoubo Jin; jin_shoubo@163.com
}

Received 13 April 2021; Revised 23 June 2021; Accepted 1 July 2021; Published 14 July 2021

Academic Editor: Siew Ann Cheong

Copyright (c) 2021 Shoubo Jin and Qiuju Yu. This is an open access article distributed under the Creative Commons Attribution License, which permits unrestricted use, distribution, and reproduction in any medium, provided the original work is properly cited.

\begin{abstract}
The leader-following consensus of linear multiagent systems with multiplicative noise and adaptive gains is investigated under the fixed directed graph. Because of multiplicative noise, the agents interacting with the leader can only obtain the inaccurate information. Firstly, this paper designs the adaptive gains of multiagent systems, which can converge to some fixed values and force the states to synchronize. Different from the mandatory gains, the adaptive gains depend on the states of agents and can be adjusted with the change of states. Secondly, it is shown that the multiagent systems without time delay can obtain mean square consensus under the adaptive gains. Moreover, in the presence of multiplicative noise and communication delay, the mean square consensus is also acquired in the same adaptive gains. Finally, the validity of the conclusion is simulated by an example.
\end{abstract}

\section{Introduction}

In recent years, the consensus of multiagent systems has been a hot topic in control theory, which can be applied to numerous fields, involving transportation networks, sensor networks, robotic networks, large population systems, smart grid, neural networks, and so forth [1-5]. The consensus is also the most basic problem of distributed cooperative control, and its core idea is to design a reasonable protocol to make all agents converge to the same target value. In the practical applications, the multiagent systems are easy to be affected by the internal structure and the external environment, so measurement noise and communication delay are inevitable $[6,7]$.

The most representative works for the consensus of multiagent systems might be the Vicsek model [8], which offered the updated methods for the position and the velocity of interacting particles at each time step and proved that the multiagent systems can achieve synchronization. Moreover, using the knowledge of graph theory and algebra, Jadbabaie et al. [1] provided a theoretical explanation of the Vicsek model and pointed out that the common quadratic
Lyapunov function is not the necessary condition for the stability of the Vicsek model. Ren and Beard [9] proved that a spanning tree is a key condition for the consensus of multiagent systems.

Time delay is widespread in the communication process. There are many reasons for time delay, such as measurement process, control elements, actuator, and so on. At present, many achievements have been made for the multiagent systems with time delay. When the time delay is less than a constant related to the maximum eigenvalue of the Laplacian matrix, Olfati-Saber and Murray [6] obtained the consensus of multiagent systems. $\mathrm{Yu}$ et al. [10] gave the necessary and sufficient conditions of the consensus for the second-order multiagent systems with time delay. The corresponding conditions were relaxed for the multiagent systems with the delayed statederivative feedback in Wu and Fang [11] and Jin et al. [12]. Moreover, many scholars have also analyzed the multiagent systems with time delay from other different aspects, including event-triggered systems, nonlinear systems, and so on $[13,14]$. However, these literatures do not consider the noise environment. 
In the real world, due to the influence of topology and external environment, random disturbance is inevitable in complex systems [15]. Specifically, the multiagent systems are usually disturbed by the white noise in the engineering control. Therefore, it is significant to study the consensus of multiagent systems in noisy environment. Generally, noise is divided into additive noise and multiplicative noise. In the multiagent systems, additive noise is normally regarded as an external disturbance and independent of the state. Huang and Manton [16] investigated the consensus of multiagent systems with additive noise and introduced the concept of mean square consensus and strong consensus. However, compared with additive noise, the multiplicative noise depends on the relative state of agents and can reflect real situation more accurately. In this case, each agent can achieve information corrupted by noise from its neighbors and leaders, and it is harder to prove the consensus of multiagent systems [17]. Recently, more and more scholars are paying attention to this issue. For the discrete integrator dynamics with multiplicative noise, Long et al. [18] analyzed the mean square consensus and the almost sure consensus. For the continuous dynamical systems, the exponential mean square consensus was acquired for the integrator systems with multiplicative noise under the fixed and switching network topologies [19]. Song et al. [20] obtained the mean square consensus in the fixed directed topology. Djaidja and $\mathrm{Wu}$ [21] generalized the corresponding conclusions to the leader-following multiagent systems with multiplicative noise. The difference between additive noise and multiplicative noise was researched in Zong et al. [22].

All the works mentioned above do not consider the adaptive gains which are dependent of their own states. Generally, the consensus gains designed by the majority of scholars are mandatory, which means that these gains are either equal to a constant or a time-varying function independent of states. However, the mandatory controller has some limitations in practical application, so we need to design the adaptive control protocol, which can adjust with the change of states. Recently, Knotek et al. [23, 24] discussed the adaptive consensus protocol with the coupling gain on directed graphs and undirected graphs, respectively. Li et al. [25] and Liang et al. [26] introduced the adaptive gain depending on the relative output information of neighboring agents. Yu et al. [27] analyzed the adaptive consensus of second-order nonlinear multiagent systems. However, their works did not consider the impact of noisy environment. Wu et al. [28] considered the adaptive bipartite consensus problem of general linear multiagent systems in noisy environment. Duan and Yang [29] discussed the adaptive consensus of multiagent systems with additive noise. Yu et al. [30] investigated the adaptive formation tracking issues for high-order nonlinear stochastic multiagent system with multiple leaders. Nevertheless, these works did not consider communication delay. To the best of the authors' knowledge, there are only a few results on the adaptive gain of multiagent systems with time delay in noisy environment. In the special case, $\mathrm{Li}$ et al. [31] analyzed flocking of multiple agents following a leader with adaptive gains. Wen et al. [32] used neural network approximate to the adaptive gains under unknown external disturbance. In this paper, the adaptive consensus of multiagent systems is investigated under multiplicative noise and communication delay. More results on the adaptive consensus can be found in Wang et al. [36-38].

In this paper, the adaptive gains are designed for the linear multiagent systems with multiplicative noise and communication delay. In the absence of communication delay, we construct a new adaptive gain and prove the mean square consensus of multiagent systems. Duan and Yang [29] analyzed the adaptive consensus of multiagent systems without communication delay under additive noise, and this paper generalizes it to multiplicative noise. Moreover, because we do not use the commutative law between a gain matrix and a positive definite matrix in Itô's differential process, the adaptive gains in this paper are different from their result, and our results are more practical. In the presence of communication delay, we investigate the mean square consensus of multiagent systems in the same adaptive gains. Although the mean square consensus of the multiagent systems with communication delay can be obtained by Lyapunov stability theorem, its preconditions are stronger than those of multiagent systems without communication delay. In this paper, the adaptive gains do not involve the delay terms, which are similar to the results in Li et al. [31] and Wen et al. [32].

There are three main contributions in this paper. (1) The adaptive gains of multiagent systems with multiplicative noise and communication delay are designed. Compared with mandatory gain, the adaptive gains can adjust with the change of states and converge to some fixed values which may not be zero. Since we do not use the commutative law between a gain matrix and a positive definite matrix in Itô's differential process, the adaptive gains in this paper are more practical. (2) No matter whether the multiagent systems have time delay or not, the mean square consensus is obtained by Lyapunov stability theorem under the same adaptive gains. However, the preconditions of the former are more complicated than those of the latter. (3) Under the same adaptive gains, the simulations show that the convergence speed of the multiagent systems without communication delay is faster than the case with communication delay.

\section{Notation and Graph Theory}

In this paper, the fixed directed graph of multiagent systems is denoted as $\mathscr{G}=(V, N, A)$, where $V=\left\{v_{1}, v_{2}, \ldots, v_{n}\right\}$ is the nonempty set of nodes, $N \subseteq V \times V$ is the set of edges, and $A=\left[a_{i j}\right] \in \mathscr{R}^{n \times n}$ is the adjacency matrix. The edge $\left(v_{i}, v_{j}\right) \in N$ signifies that agent $v_{j}$ can acquire information from agent $v_{i}$. Let $N_{i}=\left\{v_{j} \in V:\left(v_{j}, v_{i}\right) \in N\right\}$ be the set of neighbors of $v_{i}$. For a weighted digraph $\mathscr{G}$, it is defined as $a_{i i}=0$ for any $i=1,2, \ldots, n$, and $a_{i j}>0$ if $\left(v_{i}, v_{j}\right) \in N$. The Laplacian matrix of digraph can be written as $L_{\mathscr{G}}=\left[l_{i j}\right]$, where $l_{i i}=\sum_{j \neq i} a_{i j}$ and $l_{i j}=-a_{i j}, i \neq j$. A directed path from node $v_{i_{1}}$ to node $v_{i_{l}}$ is composed of a sequence of order edges, such as $\left(v_{i_{1}}, v_{i_{2}}\right),\left(v_{i_{2}}, v_{i_{3}}\right), \ldots,\left(v_{i_{l-1}}, v_{i_{l}}\right)$. 
Node $v_{i}$ to node $v_{j}$ is reachable if there is a directed path from $v_{i}$ to $v_{j}$. And node $v_{i}$ is globally reachable in digraph if there is a directed path from $v_{i}$ to any nodes.

A digraph is called balanced if all its nodes satisfy $\sum_{j=1}^{n} a_{i j}=\sum_{j=1}^{n} a_{j i}, i=1,2, \ldots, n$.

Let $\bar{G}=(\bar{V}, \bar{N}, \bar{A})$ be the fixed directed graph including nodes $v_{0}, v_{1}, v_{2}, \ldots, v_{n}$, where $v_{0}$ is the node corresponding to the leader, and $v_{1}, v_{2}, \ldots, v_{n}$ are the nodes corresponding to the followers. The relationship between the two Laplacian matrices, $\mathscr{G}$ and $\overline{\mathscr{G}}$, can be expressed as $L_{\overline{\mathscr{G}}}=$ $\left[\begin{array}{cc}0 & 0_{1 \times n} \\ -B \cdot \mathbf{1}_{n} & L_{\mathscr{G}}+B\end{array}\right]$, where $B=\operatorname{diag}\left\{a_{10}, a_{20}, \ldots, a_{n 0}\right\}$ and $\mathbf{1}_{n}=[1,1, \ldots, 1]^{T}$. Let $H=L_{\mathscr{G}}+B$, and we have the following lemma.

Lemma 1 (see [36]). The three conclusions are equivalent.

(1) Node 0 is globally reachable in the digraph $\overline{\mathscr{G}}$.

(2) $H$ is a positive stable matrix, which means that all the eigenvalues are positive.

(3) Assuming $G$ is balanced, it follows that $H+H^{T}$ is a positive definite matrix.

Notation 1. Let $R$ be the set of all real numbers and $R^{m}$ be $n$ dimensional real number space. $E[p(x)]=\int_{R^{m}} x p(x) \mathrm{d} x$ is the mathematical expectation of probability density function $p(x)$. The symbols $\underline{\theta}, \bar{\theta}, \underline{\vartheta}$, and $\bar{\vartheta}$ are positive constants.

\section{The Adaptive Consensus without Delay}

We consider the leader-following consensus of multiagent systems with multiplicative noises, which are composed of $n+1$ agents. In this paper, the subscript 0 represents the leader, and the subscripts 1 to $n$ represent the followers. The dynamics of the followers are described as

$$
\dot{x}_{i}(t)=u_{i}(t), \quad i=1,2, \ldots, n,
$$

where $x_{i}(t) \in R^{m}, u_{i}(t) \in R^{m}$ are the state and the control input of agent $i$, respectively. In this paper, the initial value $x_{i}(0)$ is finite for any $i=1,2, \ldots, n$. The leader-following controller is proposed as follows:

$$
\begin{aligned}
u_{i}(t)= & a_{i}(t)\left[\sum_{j \in N_{i}} a_{i j}\left(x_{i}(t)-x_{j}(t)\right)+a_{i 0}\left(x_{0}(t)-x_{i}(t)\right)\right. \\
& \left.+a_{i 0} \sigma_{0 i}\left(x_{0}(t)-x_{i}(t)\right) \eta(t)\right],
\end{aligned}
$$

where $a_{i}(t)$ is the consensus gain of agent $i$ such that $\underline{\theta} \leq a_{i}(t) \leq \bar{\theta}$, the constants $\underline{\theta}, \bar{\theta}>0$, and $x_{0}(t)$ represents the state of a leader and is equal to a constant in this paper. $a_{i j}$ is the element of adjacency matrix, $a_{i 0}$ indicates the weight of the information flow from the leader 0 to agent $i, \eta(t) \in R$ is one-dimensional standard white noise, that is, $\int_{0}^{t} \eta(s) \mathrm{d} s=w(t)$, where $w(t)$ is the standard Brownian motions defined on a probability space $(\Omega, \mathscr{F}, p)$, and $\sigma_{0 i} \geq 0$ represents the noise intensity. The control protocol (2) is an edge-based distributed protocol, and each agent uses only local information of its neighboring agents under the communication topology. Recently, many scholars have studied this problem. For instance, Li and Zhang [37] discussed the average-consensus control for a network of continuous-time first-order integrator agents under measurement noises. The necessary and sufficient conditions of consensus for the second-order multiagent systems were obtained under an similar protocol in Cheng et al. [38]. Djaidja and $\mathrm{Wu}$ and $\mathrm{Hu}$ and Feng $[39,40]$ analyzed the tracking control of leader-follower multiagent systems under the similar interconnection topology.

Remark 1. Protocol (2) consists of two parts. $\sum_{j \in N_{i}} a_{i j}\left(x_{i}(t)-x_{j}(t)\right)$ reflects the interaction from agent $i$ to its neighbors $j \in N_{i}$, and this part is immune to noise in this paper. The other part, $a_{0 i}\left[\left(x_{0}(t)-x_{i}(t)\right)+\right.$ $\left.\sigma_{0 i}\left(x_{0}(t)-x_{i}(t)\right)\right]$, reflects how agent $i$ communicates with the leader and includes the multiplicative noise.

In this paper, our objective is to design the adaptive consensus gains for the multiagent systems and make all the agents converge to the leader in mean square sense, i.e., $\lim _{t \rightarrow \infty} E\left|x_{i}(t)-x_{0}(t)\right|^{2}=0$ for any $i=1,2, \ldots, n$. For protocol (2), the adaptive gains are designed as

$$
\dot{a}_{i}(t)=\varepsilon(t)^{T}\left(\begin{array}{c}
p_{1 i} \\
p_{2 i} \\
\vdots \\
p_{n i}
\end{array}\right)\left(h_{i 1}, h_{i 2}, \ldots, h_{i n}\right) \varepsilon(t), \quad i=1,2, \ldots, n,
$$

where $\quad \varepsilon(t)=\left(x_{1}(t)-x_{0}(t), x_{2}(t)-x_{0}(t), \ldots, x_{n}(t)-x_{0}\right.$ $(t))^{T}$ represents the tracking error and $p_{i j}$ and $h_{i j}$ are, respectively, the elements of the matrices $P$ and $H$. The matrix $P$ is positive definite and satisfies $P H+H^{T} P=I$. After a simple calculation, adaptive gain (3) can be converted to the formula, $a_{i}(t)=\sum_{l=1}^{n} \sum_{k=1}^{n}\left(x_{k}-x_{0}\right) p_{k i} h_{i l}\left(x_{l}-x_{0}\right)$. Compared with Knotek et al. [24], the adaptive gains do not consider the decay terms that push the gains to some fixed values. Moreover, Li et al. [25] designed similar adaptive gains for the linear multiagent systems in undirected connected communication graphs. However, their conclusions did not take into account the influence of noise. Compared with [29], this paper avoids using the commutative law between a gain matrix and a positive definite matrix in Itô's differential process, so adaptive gain (3) is more practical.

Remark 2. In this paper, the adaptive gains depend on the relative states between the leader and the followers at time $t$. There have been many results when the gains are the same value and satisfy some integral conditions, i.e., $a_{i}(t)=a(t)$, $\int_{0}^{\infty} a(t) \mathrm{d} t=\infty$ and $\int_{0}^{\infty} a^{2}(t) \mathrm{d} t<\infty$. For example, $a(t)=$ $(1 / 1+t)$ and $a(t)=(\log (1+t) / 1+t)$ satisfy the conditions, and they have no ties to the state of multiagent systems. However, adaptive gains (3) can change with the tracking errors of the states. If $x_{i}(t) \longrightarrow x_{j}(t) \longrightarrow x_{0}(t)$, the consensus gain $a_{i}(t)$ in this paper will converge to an ideal value, which may not be equal to 0 . 
Remark 3. For discrete multiagent systems, the above conditions can be replaced with $\sum_{k=0}^{\infty} a(k)=\infty$ and $\sum_{k=0}^{\infty} a^{2}(k)<\infty$, and it follows that the leader-following multiagent systems can achieve mean square bounded consensus [11]. However there are few results of discrete adaptive gain $a_{i}(k)$.
Remark 4. For more general linear time invariant dynamics $\dot{x}_{i}=A x_{i}+B u_{i}$, readers can use the algebraic Riccati equation to construct other new adaptive gains, but it is not the focus of this paper.

Substituting protocol (2) into (1), it follows that

$$
\dot{x}_{i}(t)=a_{i}(t)\left[\sum_{j \in N_{i}} a_{i j}\left(x_{i}(t)-x_{j}(t)\right)+a_{i 0}\left(x_{0}(t)-x_{i}(t)\right)+a_{i 0} \sigma_{0 i}\left(x_{0}(t)-x_{i}(t)\right) \eta(t)\right] .
$$

Let $\varepsilon(t)=X(t)-1_{n} \otimes x_{0}(t)$ be the tracking error, and it can be rewritten in a compact form as

$$
\dot{X}(t)=-A(t) H X(t)+A(t) B\left(1_{n} \otimes x_{0}(t)\right)-A(t) B C\left(X(t)-1_{n} \otimes x_{0}(t)\right) \eta(t),
$$

where $A(t)=\operatorname{diag}\left\{a_{1}(t), a_{2}(t), \ldots, a_{n}(t)\right\}$ denotes the matrix of the adaptive gains, $B=\operatorname{diag}\left\{a_{10}, a_{20}, \ldots, a_{n 0}\right\}$ and $C=$ $\operatorname{diag}\left\{\sigma_{01}, \sigma_{02}, \ldots, \sigma_{0 n}\right\}$ are constant diagonal matrices, $H=$ $L_{\mathscr{G}}+B$ reflects the construction of the digraph, and $X(t)=$ $\left(x_{1}(t), x_{2}(t), \ldots, x_{n}(t)\right)^{T}$ is the state vector.

By strictly mathematical progress, we derive the following equality from $L 1_{n}=0$ :

$$
\begin{aligned}
\dot{X}(t)= & -A(t) H\left(X(t)-1_{n} \otimes x_{0}(t)\right)-A(t) H\left(1_{n} \otimes x_{0}(t)\right) \\
& +A(t) B\left(1_{n} \otimes x_{0}(t)\right)-A(t) B C \varepsilon(t) \eta(t) \\
= & -A(t) H \varepsilon(t)-A(t) B C \varepsilon(t) \eta(t) .
\end{aligned}
$$

Since the leader is constant, the tracking errors satisfy

$$
\dot{\varepsilon}(t)=-A(t) H \varepsilon(t)-A(t) B C \varepsilon(t) \eta(t) .
$$

Let $\widehat{a}_{i}(t)$ be the ideal estimation of the adaptive gain $a_{i}(t)$, and it is equal to a positive constant and satisfies $0<\underline{\vartheta} \leq \hat{a}_{i}(t) \leq \bar{\vartheta}<\infty$, where $\underline{\vartheta}$ and $\bar{\vartheta}$ are positive constants. The main result for the multiagent systems with protocol (2) and adaptive gains (3) can be expressed as follows.

Theorem 1. Assuming node 0 is globally reachable in the fixed directed topology between $n+1$ agents, the adaptive gains satisfy (3). If the corresponding parameters satisfy $\min \left((\underline{\theta}+\underline{9}) / 2 \lambda_{\max }(P), 1\right)>\bar{\theta}^{2} \max _{i=1,2, \ldots, n}\left\{\left(a_{i 0} \sigma_{0 i}\right)^{2}\right\}$, then the multiagent systems (1) with protocol (2) can asymptotically achieve mean square convergence for any infinite initial values, i.e., $\lim _{t \rightarrow \infty} E\left|x_{i}(t)-x_{0}(t)\right|^{2}=0$.

Proof. The equivalent formulation of (7) is given by the stochastic differential equations as follows:

$$
\mathrm{d} \varepsilon(t)=-A(t) H \varepsilon(t) \mathrm{d} t-A(t) B C \varepsilon(t) \mathrm{d} w(t) .
$$

Choose the Lyapunov function

$$
V(t)=V_{1}(t)+V_{2}(t)=\varepsilon(t)^{T} P \mathcal{\varepsilon}(t)+\frac{1}{2} \sum_{i=1}^{n} \widetilde{a}_{i}(t),
$$

where $\widetilde{a}_{i}(t)=a_{i}(t)-\widehat{a}_{i}(t)$. By directed calculation, we can get the value range of $V_{1}(t)$ and $V_{2}(t)$ :

$$
\lambda_{\min }(P) \mathcal{\varepsilon}(t)^{T} \varepsilon(t) \leq V_{1}(t) \leq \lambda_{\max }(P) \mathcal{\varepsilon}(t)^{T} \varepsilon(t),
$$

and $0 \leq V_{2}(t) \leq n\left(\bar{\theta}^{2}+\bar{\vartheta}^{2}\right)=\Gamma$.

Therefore, it is easy to obtain that there exists constants $m_{i}, M_{i}>0$, such that

$$
\sum_{i=1}^{n} m_{i}\left|\varepsilon_{i}(t)\right|^{2} \leq V(t) \leq \sum_{i=1}^{n} M_{i}\left|\varepsilon_{i}(t)\right|^{2} .
$$

By applying Itô formula, it holds that

$$
\mathrm{d} V(t)=\mathscr{L}_{1} V(t) \mathrm{d} t-2 \varepsilon(t)^{T} P A(t) B C \varepsilon(t) \mathrm{d} w(t),
$$

where $\quad \mathscr{L}_{1} V(t)=-2 \varepsilon \quad(t)^{T} P A(t) H \varepsilon(t)+\operatorname{tr}\left(\varepsilon(t)^{T} A(t) B\right.$ $C P A(t) B C \varepsilon(t))+\dot{V}_{2}(t)$.

Let $\widehat{A}(t)=\operatorname{diag}\left\{\widehat{a}_{1}(t), \widehat{a}_{2}(t), \ldots, \widehat{a}_{n}(t)\right\}$, and it yields 


$$
\begin{aligned}
\mathscr{L}_{1} V(t)= & -\varepsilon(t)^{T} P(A(t)+\widehat{A}(t)) H \varepsilon(t)-\varepsilon(t)^{T} P(A(t)-\widehat{A}(t)) H \varepsilon(t)+\dot{V}_{2}(t) \\
& +\operatorname{tr}\left(\varepsilon(t)^{T} A(t) B C P A(t) B C \varepsilon(t)\right) \\
\leq & -\frac{\underline{\theta}+\underline{\vartheta}}{2} \varepsilon(t)^{T}\left(P H+H^{T} P\right) \varepsilon(t)+\operatorname{tr}\left(\varepsilon(t)^{T} A(t) B C P A(t) B C \varepsilon(t)\right) \\
= & -\min \left\{\frac{\underline{\theta}+\underline{\vartheta}}{2 \lambda_{\max (P)}}, 1\right\} V(t)+\bar{\theta}^{2} \max _{i=1,2, \ldots, n}\left\{\left(a_{i 0} \sigma_{0 i}\right)^{2}\right\} V(t) \\
= & -\mu V(t),
\end{aligned}
$$

where $\quad \mu=\min \left\{(\underline{\theta}+\underline{\vartheta}) / 2 \lambda_{\max }(P), 1\right\}-\bar{\theta}^{2} \max _{i=1,2, \ldots, n}\left\{\left(a_{i 0}\right.\right.$ $\left.\left.\sigma_{0 i}\right)^{2}\right\}$. In the process of above proof, the inequality has used the adaptive gains (3) and the second equality has utilized the formula $P H+H^{\mathrm{T}} P=I$. In fact, we can derive $\dot{V}_{2}(t)=$ $\varepsilon(t)^{\mathrm{T}} P(A(t)-\widehat{A}(t)) P H \varepsilon(t)$ from the equality as follows:

$$
\begin{aligned}
& \varepsilon(t)^{T} P \widetilde{A}(t) H \varepsilon(t) \\
& =\varepsilon(t)^{T}\left(p_{i j}\right)_{n \times n} \tilde{A}(t)\left(h_{i j}\right)_{n \times n} \varepsilon(t) \\
& =\varepsilon(t)^{T}\left(\begin{array}{cccc}
\tilde{a}_{1} p_{11} & \tilde{a}_{2} p_{12} & \cdots & \tilde{a}_{n} p_{1 n} \\
\tilde{a}_{1} p_{21} & \tilde{a}_{2} p_{22} & \cdots & \tilde{a}_{n} p_{2 n} \\
& \cdots & & \\
\tilde{a}_{1} p_{n 1} & \tilde{a}_{2} p_{n 2} & \cdots & \tilde{a}_{n} p_{n n}
\end{array}\right)\left(\begin{array}{cccc}
h_{11} & h_{12} & \cdots & h_{1 n} \\
h_{21} & h_{22} & \cdots & h_{2 n} \\
& \cdots & \\
h_{n 1} & h_{n 2} & \cdots & h_{n n}
\end{array}\right) \varepsilon(t) \\
& =\varepsilon(t)^{T}\left(\begin{array}{cccc}
\sum_{i=1}^{n} \tilde{a}_{i}(t) p_{1 i} h_{i 1} & \sum_{i=1}^{n} \widetilde{a}_{i}(t) p_{1 i} h_{i 2} & \cdots & \sum_{i=1}^{n} \widetilde{a}_{i}(t) p_{1 i} h_{i n} \\
\sum_{i=1}^{n} \tilde{a}_{i}(t) p_{2 i} h_{i 1} & \sum_{i=1}^{n} \tilde{a}_{i}(t) p_{2 i} h_{i 2} & \cdots & \sum_{i=1}^{n} \widetilde{a}_{i}(t) p_{2 i} h_{i n} \\
& \ldots & & \\
\sum_{i=1}^{n} \tilde{a}_{i}(t) p_{n i} h_{i 1} & \sum_{i=1}^{n} \tilde{a}_{i}(t) p_{n i} h_{i 2} & \cdots & \sum_{i=1}^{n} \tilde{a}_{i}(t) p_{n i} h_{i n}
\end{array}\right) \varepsilon(t) \\
& =\varepsilon(t)^{T} \sum_{i=1}^{n} \widetilde{a}_{i}(t)\left(\begin{array}{cccc}
p_{1 i} h_{i 1} & p_{1 i} h_{i 2} & \cdots & p_{1 i} h_{i n} \\
p_{2 i} h_{i 1} & p_{2 i} h_{i 2} & \cdots & p_{2 i} h_{i n} \\
& \cdots & & \\
p_{n i} h_{i 1} & p_{n i} h_{i 2} & \cdots & p_{n i} h_{i n}
\end{array}\right) \varepsilon(t) \\
& =\sum_{i=1}^{n} \widetilde{a}_{i}(t) \varepsilon(t)^{T}\left(\begin{array}{c}
p_{1 i} \\
p_{2 i} \\
\vdots \\
p_{n i}
\end{array}\right)\left(h_{i 1}, h_{i 2}, \ldots, h_{i n}\right) \mathcal{E}(t) \\
& =\sum_{i=1}^{n} \widetilde{a}_{i}(t) \dot{a}_{i}(t) \\
& =\dot{V}_{2}(t) \text {, }
\end{aligned}
$$


where $\widetilde{A}(t)=A(t)-\widehat{A}(t)$.

Therefore, by integrating from $t_{0}$ to $t$ and taking mathematical expectation, we can get

$$
E V(t) \leq E\left(V\left(t_{0}\right)\right)-\mu \int_{t_{0}}^{t} E V(s) \mathrm{d} s,
$$

for any time $t>t_{0}$. Using Gronwall's inequality, we can obtain $E V(t) \leq E V\left(t_{0}\right) e^{-\mu\left(t-t_{0}\right)}$. Since the matrix $P$ is positive definite, there exists a constant $\alpha>0$ satisfying $\alpha \mathcal{E}(t)^{T} \mathcal{\varepsilon}(t) \leq \mathcal{E}(t)^{T} P \mathcal{E}(t) \leq V(t)$, and we have

$$
E\left|X(t)-1_{n} \otimes x_{0}(t)\right|^{2} \leq \frac{E V\left(t_{0}\right)}{\alpha} e^{-\mu\left(t-t_{0}\right)} .
$$

According to the known conditions, $\mu>0$, so we obtain $\lim _{t \rightarrow \infty} E\left|x_{i}(t)-x_{0}(t)\right|^{2}=0$.

\section{The Adaptive Consensus with Communication Delay}

In this section, we deal with the adaptive consensus of the multiagent systems with communication delay and multiplicative noise. For multiagent systems (1), the protocol with communication delay is described as

$$
u_{i}(t)=a_{i}(t)\left[\sum_{j \in N_{i}} a_{i j}\left(x_{i}(t-\tau)-x_{j}(t-\tau)\right)+a_{i 0}\left(x_{0}(t-\tau)-x_{i}(t-\tau)\right)+a_{i 0} \sigma_{0 i}\left(x_{0}(t-\tau)-x_{i}(t-\tau)\right) \eta(t)\right],
$$

where the constant $\tau$ is communication delay, the other notations are the same as that of Section 3, and the adaptive gains $a_{i}(t)$ satisfy (3).
Let $\varepsilon(t)=X(t)-1_{n} \otimes x_{0}(t)$; under protocol (17), multiagent systems (1) can be rewritten as

$$
\dot{X}(t)=-A(t) H X(t-\tau)+A(t) B\left(1_{n} \otimes x_{0}(t-\tau)\right)-A(t) B C \varepsilon(t-\tau) \eta(t),
$$

which is equivalent to

$$
\dot{\varepsilon}(t)=-A(t) H \varepsilon(t-\tau)-A(t) B C \varepsilon(t-\tau) \eta(t),
$$

where the error $\varepsilon(t)=X(t)-1_{n} \otimes x_{0}(t)$. For the multiagent systems with the communication delay and the adaptive gains, the main result is expressed as follows.
Theorem 2. Suppose the digraph $\overline{\mathscr{G}}$ has the same properties as Theorem 1. If there exists the constants $w_{1}, \xi>0$, such that

$$
\left\{\begin{array}{l}
\frac{\underline{\theta}+\underline{\underline{\vartheta}}}{2}>\xi \bar{\theta}^{2} \lambda_{\max }\left(P H H^{T} P\right)+w_{1} \tau+\lambda_{\max }(P) \bar{\theta}^{2} \max _{i=1,2, \ldots, n}\left\{\left(a_{i o} \sigma_{0 i}\right)^{2}\right\}, \\
\xi w_{1} \geq 2 \tau \bar{\theta}^{2}\|H\|^{2}+\bar{\theta}^{2} \max _{i=1,2, \ldots, n}\left\{\left(a_{i o} \sigma_{0 i}\right)^{2}\right\},
\end{array}\right.
$$

then multiagent systems (1) with protocol (17) and adaptive gains (3) can asymptotically achieve mean square convergence for any infinite values, i.e., $\lim _{t \rightarrow \infty} E\left|x_{i}(t)-x_{0}(t)\right|^{2}=0$.
Proof. For the problem with communication delay, the Lyapunov function can be defined as

$$
V(t)=\mathcal{\varepsilon}(t)^{T} P \mathcal{\varepsilon}(t)+w_{2} \int_{t-\tau}^{t} \mathcal{\varepsilon}(s)^{T} \mathcal{\varepsilon}(s) \mathrm{d} s+w_{1} \int_{-\tau}^{0} \int_{t+\theta}^{t}|\mathcal{E}(s-\tau)|^{2} \mathrm{~d} s+V_{2}(t),
$$

where the function is described as $V_{2}(t)=(1 / 2)$ $\sum_{i=1}^{n}\left(a_{i}(t)-\widehat{a}_{i}(t)\right)^{2}$ and the constant $w_{2}$ satisfies

$$
\frac{\underline{\theta}+\underline{\underline{\vartheta}}}{2}-\xi \bar{\theta}^{2} \lambda_{\max }\left(P H H^{T} P\right)>w_{2}>w_{1} \tau+\lambda_{\max }(P) \bar{\theta}^{2} \max _{i=1,2, \ldots, n}\left\{\left(a_{i 0} \sigma_{0 i}\right)^{2}\right\} .
$$


Using the Itô formula, we derive the stochastic delay where differential equation:

$$
\mathrm{d} V(t)=\mathscr{L}_{2} V(t) \mathrm{d} t+2 \varepsilon(t)^{T} P A(t) B C \varepsilon(t-\tau) \mathrm{d} w(t),
$$

$$
\begin{aligned}
\mathscr{L}_{2} V(t)= & -2 \varepsilon(t)^{T} P A(t) H \varepsilon(t)+2 \varepsilon(t)^{T} P A(t) H(\varepsilon(t)-\varepsilon(t-\tau)) \\
& +\operatorname{tr}\left(P A(t) B C \varepsilon(t-\tau)(A(t) B C \varepsilon(t-\tau))^{T}\right) \\
& +w_{2} \varepsilon(t)^{T} \varepsilon(t)-w_{2} \varepsilon(t-\tau)^{T} \varepsilon(t-\tau) \\
& +w_{1} \tau|\varepsilon(t-\tau)|^{2}-w_{1} \int_{t-\tau}^{t}|\varepsilon(s-\tau)|^{2} \mathrm{~d} s+\dot{V}_{2}(t) .
\end{aligned}
$$

By properties of the adaptive gains, we have

$$
\begin{aligned}
& -2 \varepsilon(t)^{T} P A(t) H \varepsilon(t)+\dot{V}_{2}(t) \\
= & -\varepsilon(t)^{T} P(A(t)+\widehat{A}(t)) H \varepsilon(t)-\varepsilon(t)^{T} P(A(t)-\widehat{A}(t)) H \varepsilon(t)+\dot{V}_{2}(t) \\
= & -\varepsilon(t)^{T} P(A(t)+\widehat{A}(t)) H \varepsilon(t) \\
\leq & -\frac{\underline{\theta}+\underline{\vartheta}}{2} \varepsilon(t)^{T}\left(P H^{T}+H^{T} P\right) \varepsilon(t) \\
= & -\frac{\underline{\theta}+\underline{\vartheta}}{2} \varepsilon(t)^{T} \varepsilon(t) .
\end{aligned}
$$

For any $\xi>0$, we obtain

$$
\begin{aligned}
& 2 \varepsilon(t)^{T} P A(t) H(\varepsilon(t)-\varepsilon(t-\tau)) \\
& \leq \xi \varepsilon(t)^{T} P A(t) H(A(t) H)^{T} P \varepsilon(t)+\xi^{-1}|\varepsilon(t)-\varepsilon(t-\tau)|^{2} \\
& \leq \xi \bar{\theta}^{2} \lambda_{\max }\left(P H H^{T} P\right) \varepsilon(t)^{T} \varepsilon(t)+\xi^{-1}|\varepsilon(t)-\varepsilon(t-\tau)|^{2} .
\end{aligned}
$$

By direct calculation, we get

$$
\begin{aligned}
& \operatorname{tr}\left(P A(t) B C \varepsilon(t-\tau)(A(t) B C \varepsilon(t-\tau))^{T}\right) \\
& \leq \lambda_{\max }(P) \bar{\theta}^{2} \max _{i=1,2, \ldots, n}\left\{\left(a_{i 0} \sigma_{0 i}\right)^{2}\right\} \varepsilon(t-\tau)^{T} \varepsilon(t-\tau) .
\end{aligned}
$$

Define the block matrix

$$
\Lambda=\left(\begin{array}{cc}
\left(\frac{\underline{\theta}+\underline{\underline{\vartheta}}}{2}-\xi \bar{\theta}^{2} \lambda_{\max }\left(P H H^{T} P\right)-w_{2}\right) I & 0 \\
0 & \left(w_{2}-w_{1} \tau-\lambda_{\max }(P) \bar{\theta}^{2} \max \left\{\left(a_{i 0} \sigma_{0 i}\right)^{2}\right\}\right) I
\end{array}\right) .
$$

Let $\chi(t)=(\varepsilon(t), \varepsilon(t-\tau))^{T}$; we derive the following inequality from (24)-(27):

$$
\mathscr{L}_{2} V(t) \leq-\chi(t)^{T} \Lambda \chi(t)+\xi^{-1}|\varepsilon(t)-\varepsilon(t-\tau)|^{2}-w_{1} \int_{t-\tau}^{t}|\mathcal{\varepsilon}(s-\tau)|^{2} \mathrm{~d} s
$$


Using the properties of integral and (19), we have

$$
\varepsilon(t)-\varepsilon(t-\tau)=\int_{t-\tau}^{t} \dot{\varepsilon}(s) \mathrm{d} s=-\int_{t-\tau}^{t} A(t) H \varepsilon(s-\tau) \mathrm{d} s-\int_{t-\tau}^{t} A(t) B C \varepsilon(s-\tau) \mathrm{d} w(t) .
$$

By the Cauchy-Schwarz inequality, we obtain

$$
E|\mathcal{E}(t)-\varepsilon(t-\tau)|^{2} \leq k \int_{t-\tau}^{t}|\varepsilon(s-\tau)|^{2} \mathrm{~d} s,
$$

where $k=2 \tau \bar{\theta}^{2}\|H\|^{2}+2 \bar{\theta}^{2} \max _{i=1,2, \ldots, n}\left\{\left(a_{i 0} \sigma_{0 i}\right)^{2}\right\}$, and $\|H\|$ is the spectral norm of the matrix $H$.

By using the known conditions, it is shown that the matrix $\Lambda$ is positive definite. Let $\lambda_{\Lambda}=\lambda_{\min }(\Lambda)$ be the minimum eigenvalue, and we get

$$
E \mathscr{L}_{2} V(t) \leq-\lambda_{\Lambda} E|\varepsilon(t)|^{2}-\lambda_{\Lambda} E|\varepsilon(t-\tau)|^{2}-\left(w_{1}-k \xi^{-1}\right) E \int_{t-\tau}^{t}|\varepsilon(s-\tau)|^{2} \mathrm{~d} s .
$$

Integrate (23) from $t_{0}$ to $t$; because $k<\xi w_{1}$ and $\lambda_{\Lambda}>0$, there exists a positive constant $\beta>0$, such that

$$
\beta E|\varepsilon(t)|^{2} \leq E V(t) \leq E V\left(t_{0}\right)+E \int_{t_{0}}^{t} \mathscr{L}_{2} V(s) \mathrm{d} s \leq E V\left(t_{0}\right)-\lambda_{\Lambda} \int_{t_{0}}^{t} E|\varepsilon(s)|^{2} \mathrm{~d} s .
$$

Taking the Gronwall inequality, we have

$$
E|\varepsilon(t)|^{2} \leq \frac{E V\left(t_{0}\right)}{\beta} \exp \left\{-\frac{\lambda_{\Lambda}}{\beta}\left(t-t_{0}\right)\right\},
$$

which implies that the multiagent systems can asymptotically achieve mean square convergence.

Remark 5. If the communication delay $\tau=0$, the assumptions of Theorem 2 will become simple, but it is slightly different from the condition of Theorem 1 . In this case, the first formula in (20) is very similar to the condition of Theorem 1 as long as $\xi=0$. However, It is worth noting that $\xi, w_{1}>0$ in (20). The second formula in (20) can easily be satisfied if $w_{1}$ is large enough and $\xi \neq 0$. In a word, the condition of Theorem 1 is simpler than that of Theorem 2 in the multiagent systems without communication.

Remark 6. Different from the existing work, the multiagent system mentioned in this paper is more complex. Firstly, the mean square consistency problem is discussed in multiplicative noise environment, which is more consistent with the real situation. Secondly, we compare the preconditions of mean square consensus of multiagent systems with and without communication delay. The mean square consensus can be achieved under the same gains regardless of communication delay, but the preconditions are more complex in the presence of communication delay, and the following simulation shows that the convergence speed of the system is slower when the communication delay is present. Finally, because this paper does not use the commutative law between a gain matrix and a positive definite matrix in Itô's differential process, the adaptive gains have a wider application than the previous work.

\section{Simulation}

In this section, the effectiveness of main theorems is simulated by an example. In a directed graph, the number 0 represents a leader, and the numbers 1,2 , and 3 represent three followers, and the interconnection topology is depicted in Figure 1. Obviously, the leader 0 is a globally reachable node. Without loss of generality, let the adjacency element $a_{i j}=1$ if $j \in N_{i}$, and $a_{i j}=0$ if $j \in N_{i}$, so the matrix $H=L_{\mathscr{G}}+B$ can be written as $H=\left[\begin{array}{ccc}2 & 0 & -1 \\ -1 & 1 & 0 \\ 0 & -1 & 1\end{array}\right]$. Using the equation $P H+H^{\mathrm{T}} P=I$, the positive definite matrix $P$ can be obtained as $P=\left[\begin{array}{lll}0.5263 & 0.5526 & 0.4211 \\ 0.5526 & 1.2368 & 0.7368 \\ 0.4211 & 0.7368 & 0.9211\end{array}\right]$. After calculation, we have the spectral radius of $H$ and the maximum eigenvalue of $P$ as $\|H\|=2.4605$ and $\lambda_{\max }(P)=2.1327$, and we can get $\lambda_{\max }\left(P H H^{T} P\right)=0.3123$.

Under multiagent systems (1)-(3) without communication delay, we take the initial states as $x_{1}(0)=-1$, $x_{2}(0)=1.8$, and $x_{3}(0)=5.8$. If the leader is fixed to $x_{0}(t)=3$, the adaptive gains' initial value is given as $a_{1}(0)=a_{2}(0)=a_{3}(0)=2$, and the noise intensities are taken as $\sigma_{01}=\sigma_{01}=\sigma_{01}=0.15$; then, the value range of the adaptive gains, $1.5 \leq a_{i}(t), \hat{a}_{i}(t) \leq 4.5$, can be obtained by numerical calculation for any $i=1,2,3$. In this case, we have 


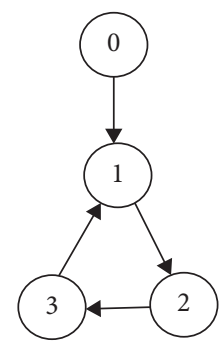

Figure 1: Digraph of the network topology.

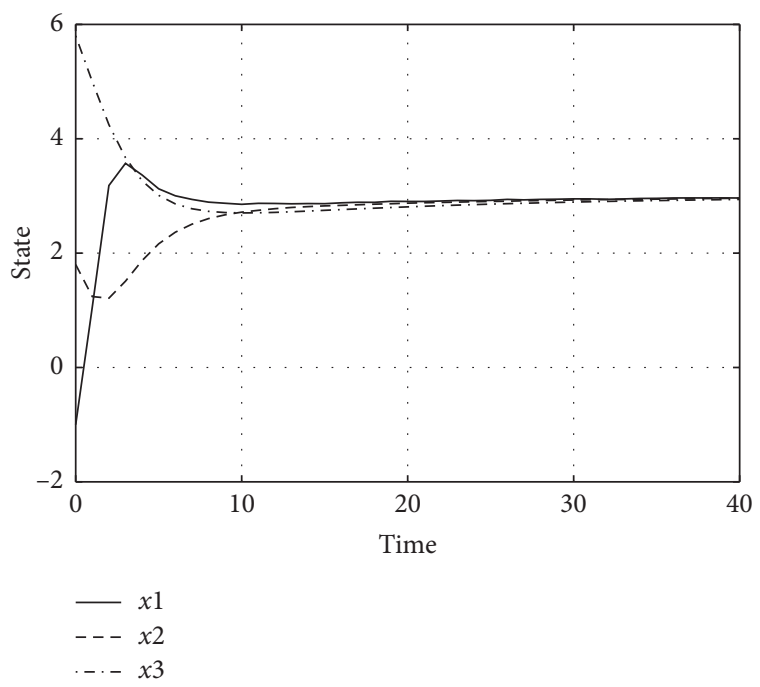

FIGURE 2: States of agents without communication delay.

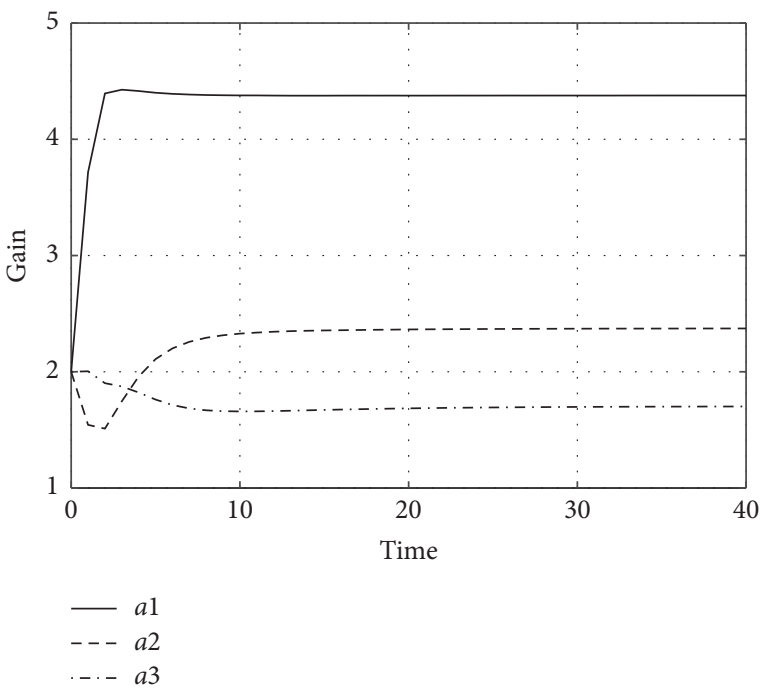

FIGURE 3: Gain trajectory of system without communication delay.

$$
\min \frac{\underline{\theta}+\underline{\vartheta}}{2 \lambda_{\max }(P), 1}=0.7033>\bar{\theta}^{2} \max _{i=1,2, \ldots, n}\left\{\left(a_{i 0} \sigma_{0 i}\right)^{2}\right\}=0.4556,
$$

which implies that the conditions of Theorem 1 hold. Setting the step length $h=0.1$, the state of agents can be simulated as

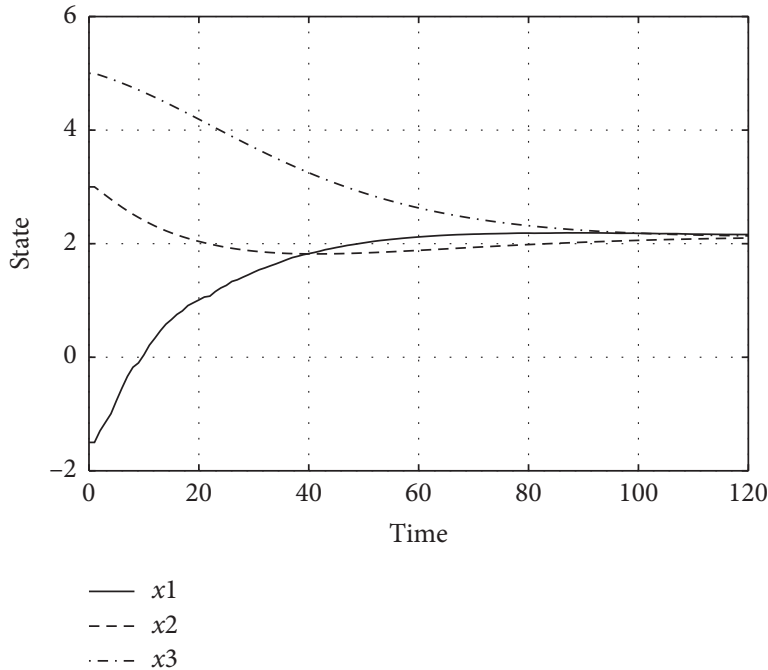

FIgURE 4: States of agents with communication delay.

shown in Figure 2, and the adaptive gains are shown by Figure 3. As can be seen from Figures 2 and 3, multiagent systems (1) with controller (2) and adaptive gains (3) can asymptotically achieve mean square convergence.

For the multiagent systems with protocol (17) and adaptive gains (3), if the initial states are $x_{1}(0)=-1.5$, $x_{2}(0)=-3$, and $x_{3}(0)=5$, the leader is taken as $x_{0}(t)=2.1$ for any $t \geq 0$. Let the communication delay $\tau=0.01$ and take the corresponding noise intensities as $\sigma_{01}=\sigma_{02}=\sigma_{03}=1$. By numerical calculation, the range of the gains can be obtained as $1.2 \leq a_{i}(t), \widehat{a}_{i}(t) \leq 2.9$. In this case, take $\xi=0.1$ and $w_{1}=20$, and we have

$$
\begin{gathered}
\frac{\underline{\theta}+\underline{\underline{\theta}}}{2}=1.2>\xi \bar{\theta}^{2} \lambda_{\max }\left(P H H^{T} P\right)+w_{1} \tau+\lambda_{\max }(P) \bar{\theta}^{2} \\
\max _{i=1,2, \ldots, n}\left\{\left(a_{i o} \sigma_{0 i}\right)^{2}\right\}=0.6420,
\end{gathered}
$$

and $\quad \xi w_{1}=2 \geq 2 \tau \bar{\theta}^{2}\|H\|^{2}+\bar{\theta}^{2} \max _{i=1,2, \ldots, n}\left\{\left(a_{i o} \sigma_{0 i}\right)^{2}\right\}=0.9342$, which imply that the conditions of Theorem 2 are satisfied. The simulation of the agents and the adaptive gains are described in Figures 4 and 5, respectively. It is easy to see from Figure 4 that the multiagent systems can asymptotically achieve mean square convergence.

The noise intensity has an important impact on the consensus of the multiagent systems with white noise. If we take the same parameters as Figure 2 and only change the noise intensity $\sigma_{0 i}$ from 0.15 to 3 , then the simulation effect of the multiagent systems (1)-(3) can be shown as Figure 6, which reveals that the dynamic evolution of the agents can produce fluctuation. In addition, Figure 7 shows that the dynamic evolution of mandatory gains is different from Figures 3 and 5 . Generally, the convergence of the gains, $a(t)=1 / 1+t$ and $a(t)=\log (1+t) / 1+t$, is mandatory, and the mandatory gains will eventually converge to zero. However, since the adaptive gains can change with the change of states, they may be able to converge to some nonzero values. 


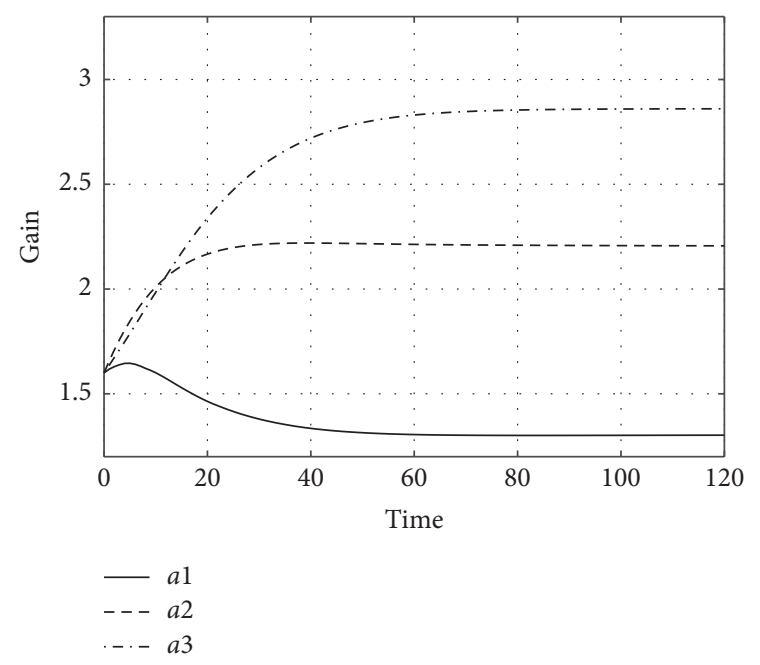

FIgURE 5: Gain trajectory of system with communication delay.

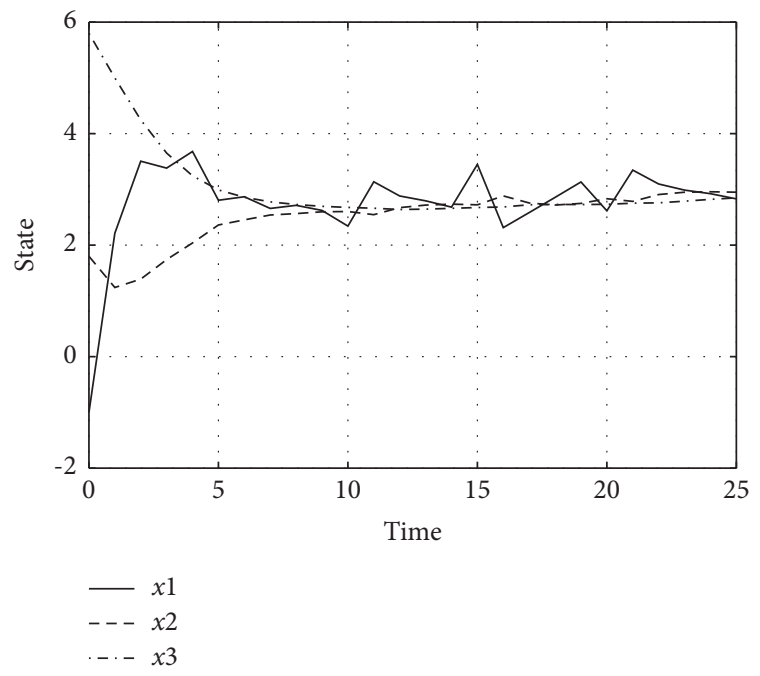

Figure 6: States of agents with large noise.

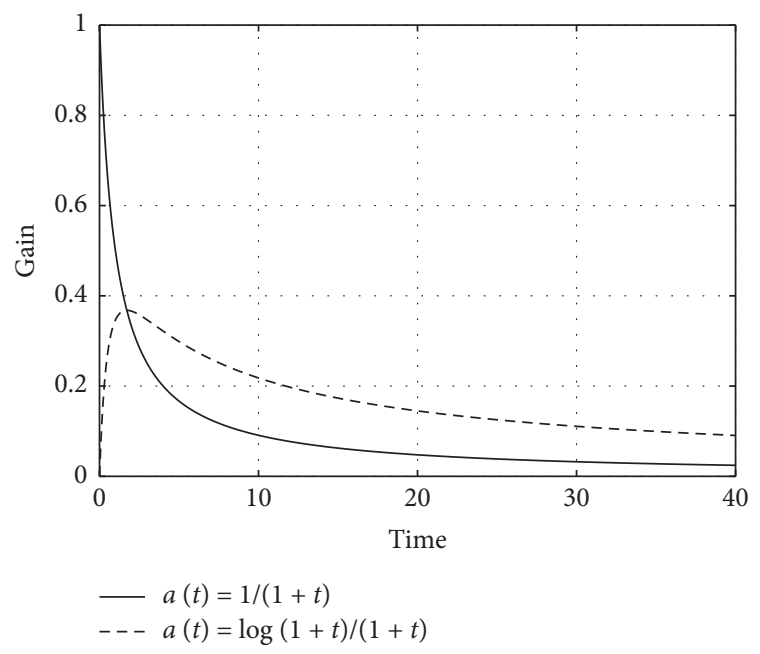

FIgURE 7: Trajectory of mandatory gain.

\section{Conclusions}

Under the fixed directed topology, this paper studies the influence of adaptive gains on the mean square consensus of multiagent systems, which is disturbed by multiplicative noise and communication delay. Firstly, we design the adaptive consensus gains based on the Lyapunov equation. Then, using the stability theory, the mean square consensus of multiagent systems is proved whether there is communication delay or not. Finally, the effectiveness of main results is simulated by an example.

\section{Data Availability}

No datasets were generated or analyzed during the current study.

\section{Conflicts of Interest}

The authors declare that they have no conflicts of interest.

\section{Acknowledgments}

This study was supported by the Provincial Natural Science Research Projects of Anhui Colleges (KJ2019A0666 and KJ2020A0735), the Key Scientific Research Projects of Suzhou University (2020yzd06), the Research Platform Projects of Suzhou University (2020ykf17), and the Quality Engineering Projects of Anhui Province (2020szsfkc0998, 2020wyxm201, and 2020jxtd286).

\section{References}

[1] A. Jadbabaie, J. Lin, and A. S. Morse, "Coordination of groups of mobile autonomous agents using nearest neighbor rules," IEEE Transactions on Automatic Control, vol. 48, no. 6, pp. 988-1001, 2003.

[2] W. Ren and Y. Gao, Communications and Control Engineering Series, Distributed Coordination of Multi-Agent Networks, Springer-Verlog, London, UK, 2007.

[3] M. Li, J. Qin, Y. Wang, and Y. Kang, "Bio-inspired dynamic collective choice in large-population systems: a robust meanfield game perspective," IEEE Transactions on Neural Networks and Learning Systems, vol. 99, pp. 1-11, 2020.

[4] R. Olfati-Saber and J. S. Shamma, "Consensus filters for sensor networks and distributed sensor fusion," in Proceedings of the 44th IEEE Conference on Decision and Control, pp. 6698-6703, Seville, Spain, December 2005.

[5] W. F. Fu, J. H. Qin, Y. Shi et al., "Resilient consensus of discrete-time complex cyber-physical networks under deception attacks," IEEE Transactions on Industrial Informatics, vol. 16 , no. 7, pp. 4868-4877, 2020.

[6] R. Olfati-Saber and R. M. Murray, "Consensus problems in networks of agents with switching topology and time-delays," IEEE Transactions on Automatic Control, vol. 49, no. 9, pp. 1520-1533, 2004.

[7] T. C. Asya and K. E. Barner, "Convergence of consensus models with stochastic disturbances," IEEE Transaction on Information Theory, vol. 56, pp. 4101-4113, 2010.

[8] T. Vicsek, A. Czirok, E. Ben-Jacob et al., "Novel type of phase transition in a system of self-driven particles," Physical Review Letters, vol. 75, no. 6, pp. 1226-1229, 2006. 
[9] W. Ren and R. W. Beard, "Consensus seeking in multiagent systems under dynamically changing interaction topologies," IEEE Transactions on Automatic Control, vol. 50, no. 5, pp. 655-661, 2005.

[10] W. Yu, G. Chen, and M. Cao, "Some necessary and sufficient conditions for second-order consensus in multi-agent dynamical systems," Automatica, vol. 46, no. 6, pp. 1089-1095, 2010.

[11] Z. Wu and H. Fang, "Delayed-state-derivative feedback for improving consensus performance of second-order delayed multi-agent systems," International Journal of Systems Science, vol. 43, no. 1, pp. 140-152, 2012.

[12] S. B. Jin, Y. H. Li, and Z. H. Wei, "Research on control of protocol of multi-agent system based on large communication delay," Journal of Systems Science and Mathematical Sciences (A), vol. 40, no. 9, pp. 1531-1538, 2020.

[13] C. Deng, M. J. Er, G.-H. Yang et al., "Event-triggered consensus of linear multi-agent system with time-varing communication delays," IEEE Transactions on Cybernetics, vol. 50, no. 7, pp. 2916-2925, 2020.

[14] J. Q. Lu, X. Guo, T. W. Huang et al., "Consensus of signed network multi-agent systems with nonlinear coupling and communication delays," Applied Mathematics and Computation, vol. 35, pp. 153-162, 2019.

[15] X. Mao, Stochastic Differential Equations and Their Applications, Horwood Publishing Limited, Chichester, UK, 1997.

[16] M. Y. Huang and J. H. Manton, "Coordination and consensus of networked agents with noisy measurements, stochastic algorithms and asymptotic behavior," SIAM Journal on Control and Optimization, vol. 48, no. 1, pp. 134-161, 2009.

[17] V. P. Zuzlukov, Signal Processing Noise, CRC Press, Roca Raton, FL, USA, 2002.

[18] Y. S. Long, S. Liu, and L. H. Xie, "Distributed consensus of discrete-time multi-agent systems with multiplicative noise," International Journal of Robust and Nonlinear Control, vol. 25, no. 16, pp. 3113-3131, 2015.

[19] Y. H. Ni and X. Li, "Consensus seeking in multi-agent systems with multiplicative measurement," System and Control Letters, vol. 62, pp. 430-437, 2013.

[20] L. Song, D. Huang, S. K. Nguang et al., "Mean square consensus of multi-agent systems with multiplicative noises and time delays under directed fixed topologies," International Journal of Control, Automation and Systems, vol. 14, no. 1, pp. 69-77, 2016.

[21] S. Djaidja and Q. H. Wu, "Leader-following consensus of single-integrator multi-agent systems under noisy and delayed communication," International Journal of Control, Automation and Systems, vol. 14, no. 2, pp. 357-366, 2016.

[22] X. F. Zong, T. Li, and J. F. Zhang, "Consensus conditions of continuous-time multi-agent systems with time-delays and measurement noises," Automatica, vol. 99, pp. 412-419, 2019.

[23] T. Knotek, K. Hengster-Morric, and M. Ebek, "Distributed adaptive consensus protocol with decaying gains on directed graphs," IFAC-papers Online, vol. 49, no. 22, pp. 355-360, 2016.

[24] T. Knotek, K. Hengster-Morric, and M. Ebek, "Distributed adaptive consensus protocol with decaying gains," International Journal of Robust and Nonlinear Control, vol. 30, no. 15, pp. 6166-6188, 2020.

[25] Z. K. Li, W. Ren, X. D. Liu et al., "Distributed consensus of linear multi-agent Systems with adaptive dynamic protocols," Automatica, vol. 49, no. 7, pp. 1786-1995, 2013.

[26] Q. P. Liang, Y. Z. Wu, J. P. Hu et al., "Bipartite output synchronization of heterogeneous time-varying multi-agent systems via edge-based adaptive protocols," Journal of the Franklin Institute, vol. 357, no. 17, pp. 12808-12824, 2020.

[27] Z. Y. Yu, D. Huang, H. J. Jiang et al., "Consensus of secondorder multi-agent systems with nonlinear dynamics via edgebased distributed adaptive protocols," Journal of the Franklin Institute, vol. 353, pp. 4821-4844, 2016.

[28] Y. Z. Wu, Q. P. Liang, Y. Y. Zhao et al., "Adaptive bipartite consensus control of general linear multi-agent systems using noise measurments," European Journal of Control, vol. 59, pp. 123-128, 2021.

[29] Y. B. Duan and Z. W. Yang, "Design and analysis of the consensus gain for stochastic multi-agent systems," Control Theory and Application, vol. 36, no. 4, pp. 629-635, 2019.

[30] J. L. Yu, X. W. Dong, Q. D. Li et al., "Fully adaptive practical time-varying output formation tracking for high-order nonlinear stochastic multiagent system with multiple leaders," IEEE Transaction on Cybernetics, vol. 57, no. 4, pp. 2265-2277, 2021.

[31] S. K. Li, X. Z. Liu, W. S. Tang et al., "Flocking of multi-agents following a leader with adaptive protocol in a noisy environment," Asian Journal of Control, vol. 16, no. 6, pp. 1771-1778, 2014.

[32] G. X. Wen, L. L. Philip Chen, Y. J. Liu et al., "Neural networkbased adaptive leader-following consensus control for a class of nonlinear multi-agent state-delay systems," IEEE Transaction on Cybernetics, vol. 47, no. 8, pp. 2151-2160, 2017.

[33] Q. L. Wang and C. Y. Sun, "Adaptive consensus of multiagent systems with unknown high-frequency gain signs under directed graphs," IEEE Transactions on Systems, Man and Cybernetics Systems, vol. 50, no. 6, pp. 2151-2186, 2020.

[34] A. u. Rehman, M. Rehan, N. Iqlah et al., "Leaderless adaptive output feedback consensus approach for oneside Lipschitz multi-agents," Journal of the Franklin Institute, vol. 357, pp. 8800-8822, 2020.

[35] H. Q. Pei and Q. Lai, "Consensus of second-order multiagent systems with directed signed networks and communication delays," Complexity, vol. 2020, Article ID 1712643, 10 pages, 2020.

[36] J. P. Hu and Y. G. Hong, "Leader-following coordination of multi-agent system with coupling time delays," Physica A, Statistical Mechanics and Its Applications, vol. 374, no. 2, pp. 853-863, 2007.

[37] T. Li and J. F. Zhang, "Mean square average-consensus under measurement noises and fixed topologies: necessary and sufficient conditions," Automatica, vol. 45, pp. 1929-1936, 2009.

[38] L. Cheng, Z. G. Hou, M. Tang et al., "Necessary and sufficient conditions for consensus of double-integrator multi-agent systems with measurement noises," IEEE Transactions on Automatic Control, vol. 56, no. 8, pp. 1958-1963, 2011.

[39] S. Djaidja and Q. H. Wu, "Stochastic consensus of leaderfollowing multi-agent systems under additive measurement noises and time-delays," European Journal of Control, vol. 23, pp. 55-61, 2015.

[40] J. P. Hu and G. Feng, "Distributed tracking control of leaderfollower multi-agent systems under noisy measurement," Automatica, vol. 46, no. 8, pp. 1382-1387, 2010. 